Xuefeng Jiang, Qin Ouyang*, Dapeng Liu, Jing Huang, Hongbo Ma, Yousi Chen, Xuefei Wang and Wei Sun

\title{
Preparation of low-cost carbon fiber precursors from blends of wheat straw lignin and commercial textile-grade polyacrylonitrile (PAN)
}

https://doi.org/10.1515/hf-2017-0191

Received November 22, 2017; accepted April 23, 2018; previously published online May 28, 2018

\begin{abstract}
Low-cost precursor fibers (PFs) were prepared from blends of a wheat straw lignin (WSL) and a commercial textile-grade polyacrylonitrile (PAN) by wet spinning, and then the precursors were converted into carbon fibers (CFs) by thermal stabilization and carbonization. The PFs were characterized by scanning electron microscopy (SEM), Fourier-transform infrared spectroscopy (FTIR) and differential scanning calorimetry (DSC). The lignin content in the blends was found to play an important role in the PF structure, which was closely related to the change of intermolecular forces in the spinning solution. The lignin, acting as the carboxylic acid comonomer, had significantly promoted the thermal stabilization of the lignin/PAN blend PFs, which helped to further decrease the production cost of CFs. With increasing lignin contents, the carbon content of CFs remained at about $95 \%$. The carbon of lignin could be utilized for the preparation of CFs.
\end{abstract}

Keywords: blend, carbon fiber, lignin, polyacrylonitrile, precursor, thermal stabilization, wet spinning

\footnotetext{
*Corresponding author: Qin Ouyang, National Engineering Laboratory for Carbon Fiber Preparation Technology, Ningbo Institute of Materials Technology and Engineering, Chinese Academy of Sciences, Ningbo 315201, China; and Key Laboratory of Bio-based Polymeric Materials Technology and Application of Zhejiang Province, Ningbo 315201, China, e-mail: o_yang@126.com, ouyangqin@nimte.ac.cn

Xuefeng Jiang: National Engineering Laboratory for Carbon Fiber Preparation Technology, Ningbo Institute of Materials Technology and Engineering, Chinese Academy of Sciences, Ningbo 315201, China; and College of Material Science and Chemical Engineering, Ningbo University, Ningbo 315211, China

Dapeng Liu, Jing Huang, Hongbo Ma, Yousi Chen and Xuefei Wang National Engineering Laboratory for Carbon Fiber Preparation Technology, Ningbo Institute of Materials Technology and Engineering, Chinese Academy of Sciences, Ningbo 315201, China Wei Sun: College of Material Science and Chemical Engineering, Ningbo University, Ningbo 315211, China
}

\section{Introduction}

Carbon fibers (CFs) have attracted attention worldwide as strong and lightweight reinforcement materials for fabricating advanced composites (Huang 2009). Currently, more than $90 \%$ of commercial CFs are produced from polyacrylonitrile (PAN). Due to their excellent mechanical properties, PAN-CFs have been widely applied in aviation, aerospace and high-end sporting goods industries (Xia et al. 2016). However, the high price of the petroleumderived raw material and the high-cost production process have limited the applications of PAN-CFs in more common areas including automotive and construction industries (Foston et al. 2013).

The precursor fibers (PFs) contribute about half of the total cost of the conventional PAN-CFs (Warren et al. 2009). Therefore, lowering the cost of PF production is important and there are various ways to reach this goal. For example, the textile-grade PAN-PFs have been used for CF production (Yoo et al. 2017), new melt-spinnable PAN-PFs were synthesized (Miller et al. 2017) and polyolefin and lignin were converted to CFs (Chatterjee and Saito 2015). Among all these candidates, lignin is meaningful and of great importance, because it is an abundant carbon-rich renewable resource, available as a major by-product of the pulping industry (Wang et al. 2015). Technical lignins have recently attracted great attention as activated CFs for carbon yarn supercapacitors (Huang and Zhao 2016; Huang et al. 2018) or for multifunctional lithium-ion battery electrodes (Nowak et al. 2018).

Lignin is a complex amorphous polymer with a crosslinked network structure (Ye et al. 2017), which results in poor spinnability. The development of continuous lignin PFs is a great challenge (Mainka et al. 2015) and various methods have been proposed to this purpose (Youe et al. 2016; Park et al. 2017). A usual approach is to blend lignin with other polymers with good spinnability. Some thermoplastic polymers, such as poly(ethylene oxide) (PEO), poly(ethylene terephthalate) (PET), polypropylene (PP) and poly(lactic acid) (PLA), were tested to prepare lignin PFs by melt spinning (Ragauskas et al. 2014). However, the poor 
thermal stability and poor miscibility of these polymers led to unacceptable mechanical properties of the $\mathrm{CFs}$.

PAN is also a good candidate for blending with lignin to prepare PFs (Seydibeyoglu 2012). Continuous PFs can be obtained from a lignin/PAN blend by conventional wet spinning as described by Husman (2012), while CFs with a tensile strength and a tensile modulus of about $2.24 \mathrm{GPa}$ and $217 \mathrm{GPa}$, respectively, were obtained (Husman 2014). However, when lignin contents are above $20 \%$, unacceptable macro-voids are formed in the CFs, which was also observed by Dong et al. (2015). High molecular weight (MW) PAN for blending lignin is one of the remedies for avoiding macro-void formation via increasing the viscosity of the spinning solution (Thunga et al. 2014). One problem is the homogeneous dissolution of the high MW PAN in the spinning solution. Moreover, the high production cost of such CFs is also a challenge.

Liu et al. (2015) reported a novel gel-spinning technique for preparing lignin/PAN blend $\mathrm{PFs}$ with $-50^{\circ} \mathrm{C}$ methanol as a coagulation bath, i.e. the coagulated fibers were stored in a methanol bath at $-50^{\circ} \mathrm{C}$ for over $12 \mathrm{~h}$ and then the fibers were drawn with a ratio of 13 . The gel-spun PAN/lignin (70/30)-based CFs exhibit a tensile strength of $1.72 \mathrm{GPa}$ and a tensile modulus of $230 \mathrm{GPa}$, and there are no voids observable in the cross-sections of the fiber (Liu et al. 2015). However, a technical challenge of this approach is the harsh process condition.

In the present study, a wheat straw lignin (WSL) was blended with a commercial textile-grade PAN polymer for PFs. This PAN is cheap and has a good solubility. Continuous PFs were prepared from the blends by a conventional wet spinning process and then converted into PFs via thermal stabilization and carbonization. The PFs were characterized by scanning electron microscopy (SEM), Fourier-transform infrared (FTIR) spectroscopy and differential scanning calorimetry (DSC). The stabilization of the PFs and the structure and composition of the final CFs are addressed.

\section{Materials and methods}

A WSL was provided by Anhui Geyi Co., Ltd. (China) and used after purification by desalting with diluted $\mathrm{HCl}$. A commercial textile-grade PAN polymer powder (provided by Zhejiang Hangzhouwan Co., Ltd., China) and dimethyl sulfoxide (DMSO) (from Sinopharm Chemical Reagent Co., Ltd., China) were used as received. Deionized water was produced in our own laboratory.

A series of spinning solutions were prepared by blending different ratios of the WSL and PAN polymer in DMSO (for composition see Table 1). The ratio of PAN/DMSO was fixed to $20 / 80$ by weight, but the WSL content in the blends varied from $0,5,15,20,30$ to $40 \%$; and the solid content of the spinning solution increased correspondingly from $20,20.8,22.7,23.8,26.3$ to $29.4 \%$.
Table 1: Composition of the spinning solutions.

\begin{tabular}{lrrrrrr}
\hline & & \multicolumn{5}{c}{ Lignin content in solution (\%) } \\
\cline { 2 - 7 } Materials & $\mathbf{0}$ & $\mathbf{5}$ & $\mathbf{1 5}$ & $\mathbf{2 0}$ & $\mathbf{3 0}$ & $\mathbf{4 0}$ \\
\hline Lignin (g) & 0 & 1 & 3.53 & 5 & 8.6 & 13.3 \\
PAN (g) & 20 & 20 & 20 & 20 & 20 & 20 \\
DMSO (g) & 80 & 80 & 80 & 80 & 80 & 80 \\
Total (\%) & 20 & 20.8 & 22.7 & 23.8 & 26.3 & 29.4 \\
\hline
\end{tabular}

Lignin and PAN were dissolved in DMSO at $60^{\circ} \mathrm{C}$ for $24 \mathrm{~h}$ under continuous agitation, and then deaerated under static condition at $60^{\circ} \mathrm{C}$ for $12 \mathrm{~h}$. The spinning solution was extruded through a spinneret ( 50 holes, $0.1 \mathrm{~mm}$ diameter) into a water coagulation bath. The total stretch ratios were 3 .

The PFs were placed into an air furnace and heated from 200 to $250^{\circ} \mathrm{C}$ at a heating rate of $1{ }^{\circ} \mathrm{C} \mathrm{min}^{-1}$, and the final temperature was maintained for $0.5 \mathrm{~h}$. The thermo-stabilized fibers were carbonized under nitrogen atmosphere by heating from room temperature to $700^{\circ} \mathrm{C}$, and the final temperature was maintained for $20 \mathrm{~min}$, and then heated to $1400^{\circ} \mathrm{C}$, and maintained at this temperature for $10 \mathrm{~min}$. The heating rate was $10^{\circ} \mathrm{C} \mathrm{min}^{-1}$.

The viscosity of the spinning solutions was tested by a DV-II+Pro rotational rheometer of the Brookfield Company (USA) at $60^{\circ} \mathrm{C}$ at a rotation rate of $5 \mathrm{rpm}$. The number average molecular weight (Mn) and weight average molecular weight $(\mathrm{Mw})$ data were obtained by gel permeation chromatography (GPC) (TOSOH HLC-8320, Japan) equipped with a refractive index (RI) detector, and then the dispersivity $(\mathrm{Mw} / \mathrm{Mn})$ was calculated. The mobile phase was $0.01 \mathrm{~mol} \mathrm{l}^{-1}$ $\mathrm{LiBr} /$ dimethylformamide (DMF) $\left(40^{\circ} \mathrm{C}\right)$.

FTIR spectra were recorded on a Thermo Nicolet Nexus 470 FTIR spectrometer using the $\mathrm{KBr}$ pellet technique (32 scans at a resolution of $4 \mathrm{~cm}^{-1}$ ). DSC analysis was carried out on a NETZSCH STA 449 thermal analyzer under air atmosphere at a rate of $10^{\circ} \mathrm{C} \mathrm{min}-1$ from room temperature to $500^{\circ} \mathrm{C}$. SEM images of the PFs and CFs were produced by a Hitachi S-4800 field-emission scanning electron microscope (FEM), while the samples were sputter-coated with gold before imaging. The average fiber diameter was obtained by measuring 10 fibers randomly on the SEM images.

\section{Results and discussion}

PAN exhibits a sharp and narrow GPC profile in shorter retention times, while the lignin GPC profile is broad at longer retention times (Figure 1a). Accordingly, lignin is more polydisperse, which causes the known poor spinnability (Li et al. 2017). On the other hand, PAN has a higher MW and a more uniform MW distribution (MWD) (Table 2). PAN is a linear long-chain polymer and predestined for improving the lignin's spinnability in lignin/PAN blends.

The IR band of PAN (Figure $1 \mathrm{~b}$ ) at $2243 \mathrm{~cm}^{-1}$ is assigned to $\mathrm{C} \equiv \mathrm{N}$ stretching vibration in PAN unit. The band at about $1736 \mathrm{~cm}^{-1}$ is due to the $\mathrm{C}=0$ stretching vibration of vinyl acetate (VAc). The bands of the stretching and bending 


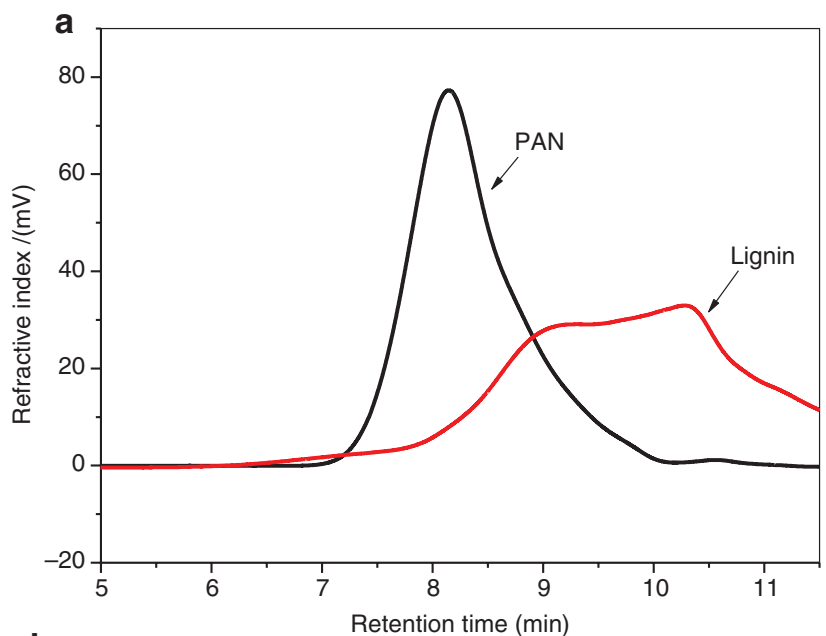

$$
\text { b }
$$

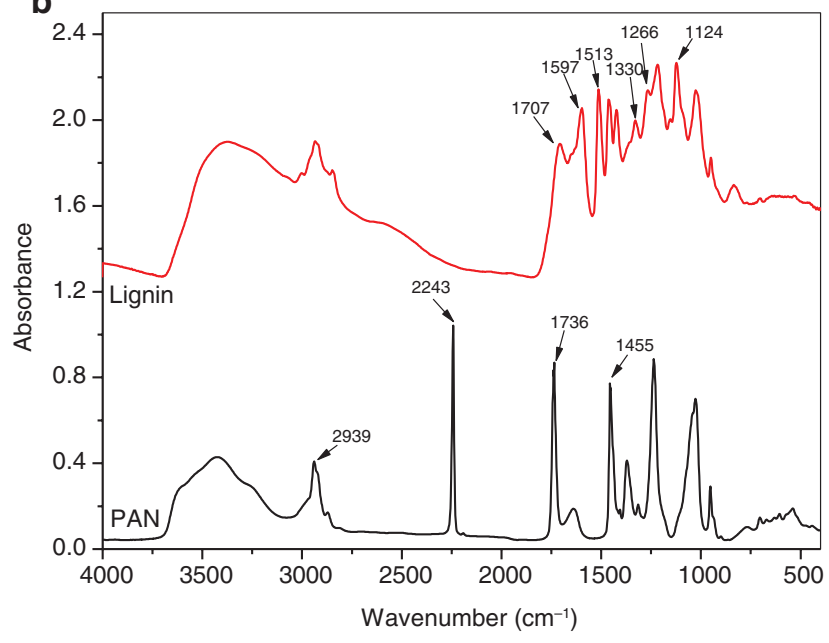

Figure 1: (a) GPC curves and (b) FTIR spectra of lignin and PAN.

Table 2: Molecular weight and its distribution of PAN and lignin.

\begin{tabular}{lrrr}
\hline & Mn & Mw & Mw/Mn \\
\hline PAN & $7.1 \times 10^{4}$ & $33.9 \times 10^{4}$ & 4.8 \\
Lignin & 112 & $7.7 \times 10^{4}$ & 690.6 \\
\hline
\end{tabular}

vibration of $\mathrm{CH}_{2}$ are observed at 2939 and $1455 \mathrm{~cm}^{-1}$, respectively. The bands at 1423, 1513 and $1597 \mathrm{~cm}^{-1}$ originate from the lignin's aromatic skeletal vibrations, and the aromatic ring vibration is seen at $1462 \mathrm{~cm}^{-1}$. The appearance of the bands in the range of 1266 to $1330 \mathrm{~cm}^{-1}$ is typical for guaiacyl and syringyl groups in lignin (Boeriu et al. 2004). The band at $1707 \mathrm{~cm}^{-1}$ is due to carbonyl/carboxyl groups.

As is visible in Figure 2a, the viscosity of the spinning solutions first increases gradually with increasing lignin content in the blends, which is highest at 30\% lignin content. Interestingly, the viscosity decreases again at lignin contentrations $>30 \%$. The viscosity of concentrated polymer
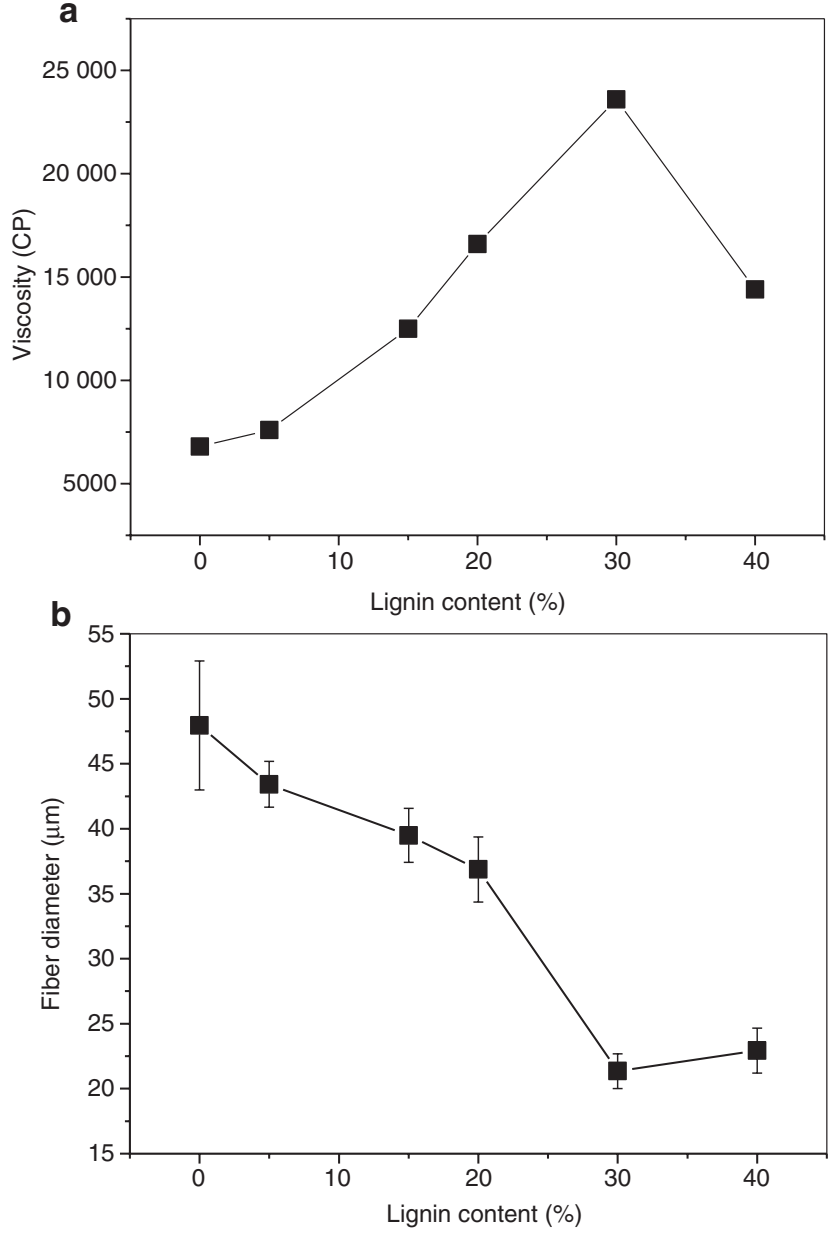

Figure 2: (a) Viscosity of the spinning solutions and (b) the diameter of PFs.

solutions is due to the internal friction of the polymer molecules (Shan et al. 2009), which is caused by the intermolecular forces between them (Doi and Edwards 1978). In the spinning solution in focus, PAN molecules are dispersed in the solution in the form of random coils, which are twisted and form a network structure, while the PAN-PAN intermolecular forces on the knots contribute primarily to viscosity. Below 30\% lignin concentration, the increased PAN-lignin intermolecular forces contribute mainly to the increase of the solution's viscosity. However, at higher lignin concentrations, the weak lignin-lignin intermolecular forces begin to dominate, and, as a consequence, the viscosity decreases. The lignin molecules increasingly fill the space between the network of PAN molecules, which will be then more isolated from each other, and thus the strong PAN-PAN intermolecular forces are destroyed. Because of the low MW of lignin, the viscosity of the spinning solution decreases again.

The diameters of the PFs with different lignin contents are presented in Figure 2b. The fiber diameters first 
decrease with increasing lignin contents because of the first viscosity increment (Figure 2a). A higher viscous solution extrudes out of the spinneret at a lower rate and this results in smaller fiber diameters. On the other hand, at lignin concentrations $>40 \%$, i.e. at lower viscosities, the fiber diameters increase correspondingly.

In the photograph of the PFs in Figure 3a, the pure PAN-PFs are white; while the lignin/PAN blend PFs are brown. The color of the blend gradually deepens with increasing lignin contents. As is visible on the SEM images in Figure 3b, all the PFs have a noncircular cross-section, which is mainly because of the poor hydrophilicity of the commercial textile-grade PAN polymer. In addition, a pure water coagulation bath was used for fiber spinning resulting in a rapid counter diffusion rate due to the large concentration difference between the spinning solution and the coagulation bath. The fiber epidermis was solidified fast before the solidification of the fiber's interior. As

a

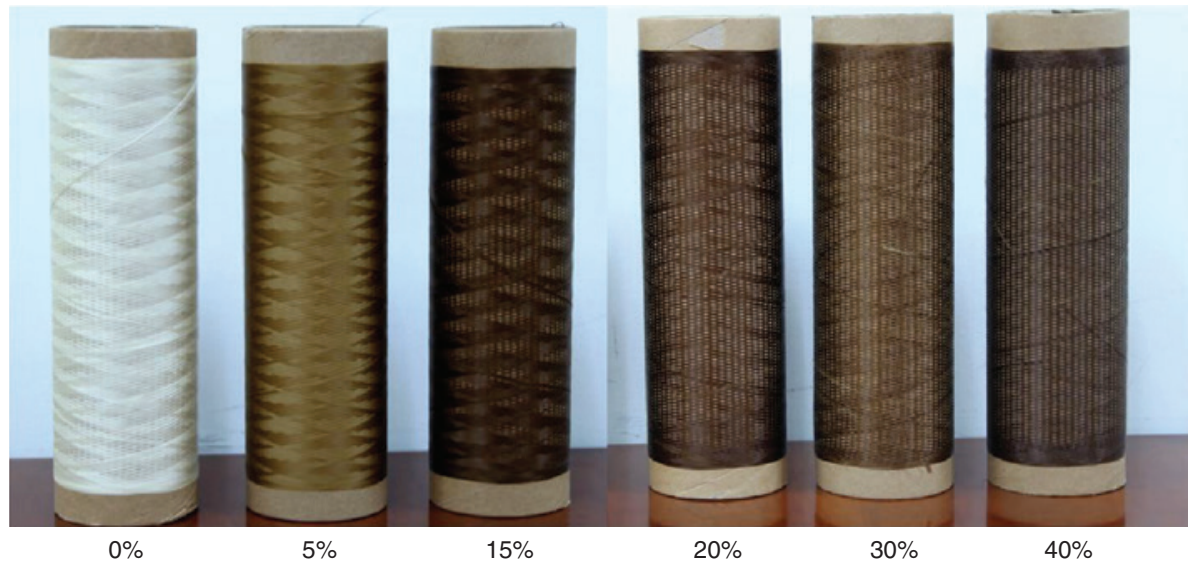

b

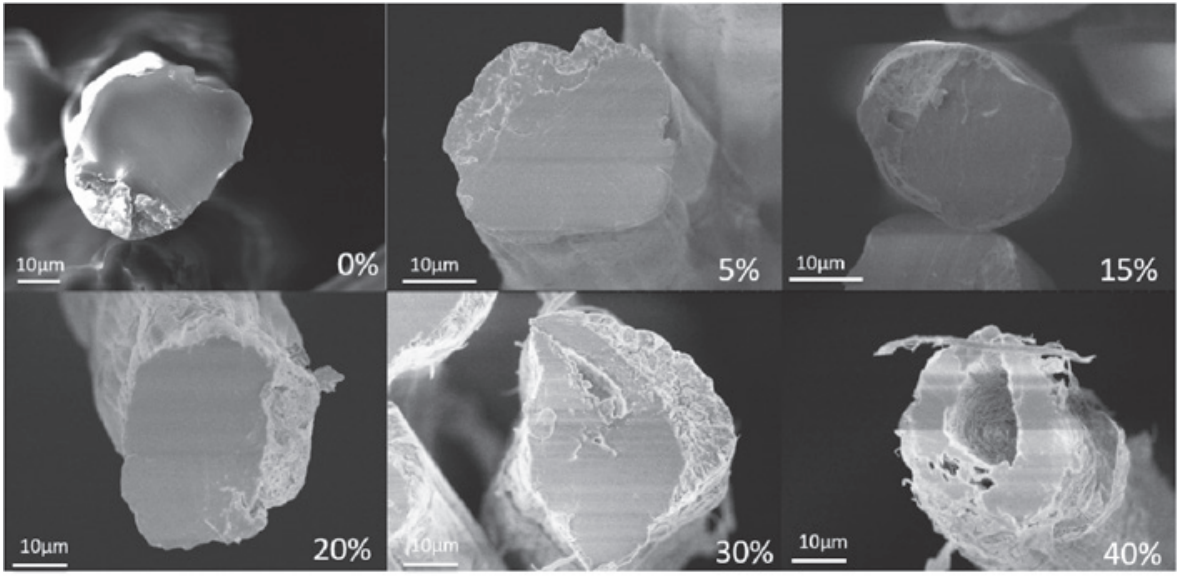

C

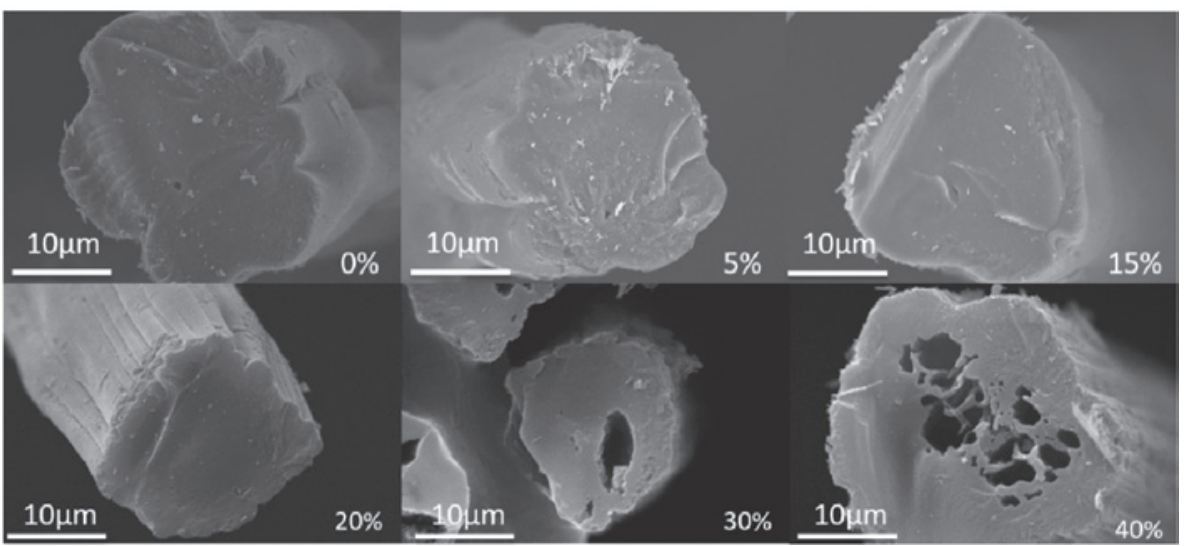

Figure 3: (a) Photograph and (b) SEM images of PFs, and (c) SEM images of CFs. 
a result, a heterogeneous radial structure was formed, which shrunk unevenly during stretching and drying, leading to noncircular cross-sections. Below 20\% lignin concentration, the PFs have a dense structrue without any visible macro-voids (Figure $3 \mathrm{~b}$ ). But around 30 and $40 \%$ lignin contents, macro-voids appear in the center of the PFs. The morphology of the CFs is inherited from the PFs (Figure 3c), and both of them represent the corresponding morphologies.

The solid content of the spinning solution increased with increasing lignin contents (Table 1). The macro-voids are a manifestation of phase separation between lignin and PAN because of their different hydrophilicity. Lignin contains more hydrophylic $\mathrm{OH}$ groups with the tendency to form intermolecular hydrogen bonds, which leads to a single phase between lignin molecules. On the other hand, the intermolecular dipole-dipole interaction among the PAN body also leads to the formation of another single phase due to strong polar nitrile groups. It is also obvious that the high MW PAN form more tight structures than the low MW lignin, which also contributes to phase separation.

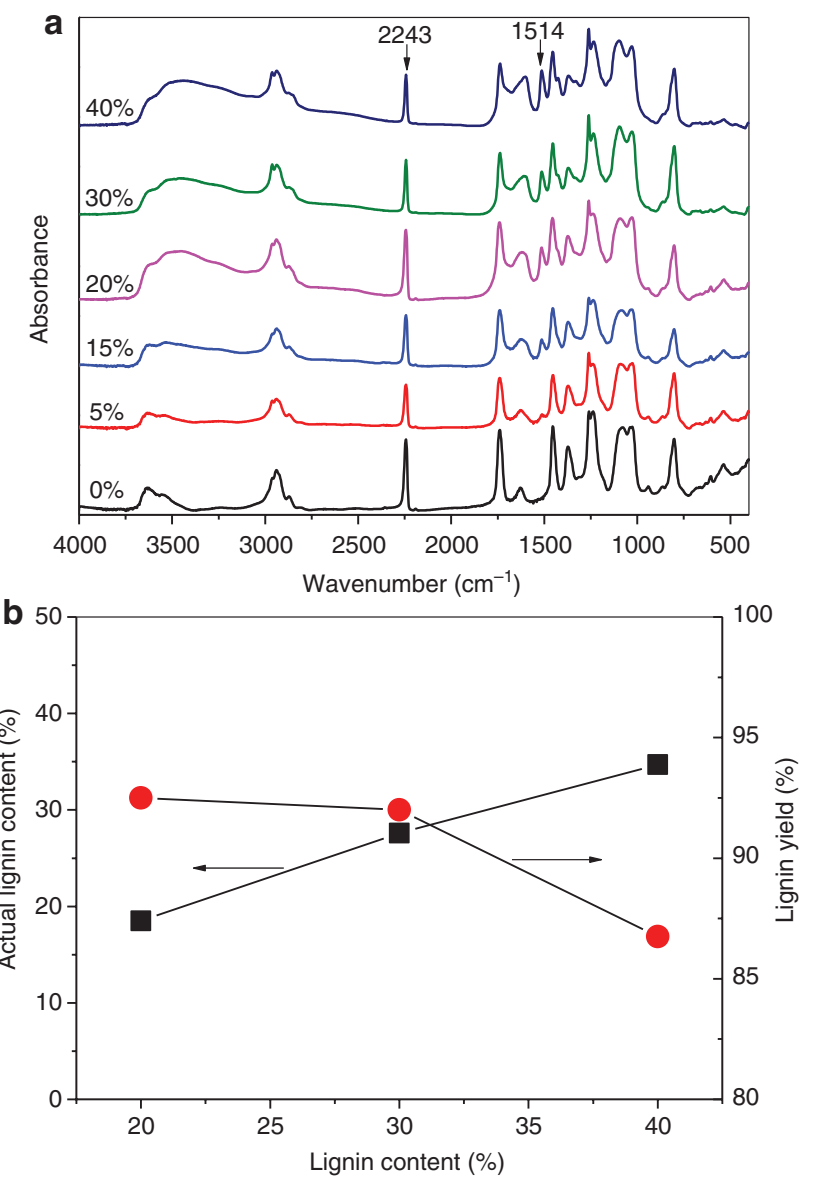

Figure 4: (a) FTIR spectra and (b) lignin content of PFs.
Figure $4 a$ shows the FTIR spectra of PFs. The $2243 \mathrm{~cm}^{-1}$ band corresponds to the $\mathrm{C} \equiv \mathrm{N}$ group of $\mathrm{PAN}$; the $1513 \mathrm{~cm}^{-1}$ band is for the aromatic ring of lignin. The spectra show that lignin and PAN are a simple mixture without chemical bonds between them. However, with increasing lignin contents, the $2243 \mathrm{~cm}^{-1}$ band ( $\mathrm{C} \equiv \mathrm{N}$ group of PAN) decreases and the $1513 \mathrm{~cm}^{-1}$ band (aromatic ring of lignin) increases gradually. Thus, the relative lignin content of the PFs can be calculated based on the band ratios $\mathrm{A}_{1513} / \mathrm{A}_{2243^{\circ}}$. The corresponding calibration plots are presented in Figure $\mathrm{S} 2$ in the Supplementary Material.

The actual lignin content and the lignin yield of the lignin/PAN blend PFs are presented in Figure 4b, which are somewhat lower than the lignin content in the blend spinning solutions. This is because lignin was partially washed out during spinning. When the lignin content in the spinning solutions is between 20 and 30\%, the lignin yield in the PF is about $92 \%$. At higher lignin concentrations, the lignin yield decreases to values around $87 \%$.

The DSC curves (Figure 5) of PAN exhibits a strong exothermic maximum, which is attributed to both the cyclization and oxidation reactions (Arbab et al. 2014). The initiation temperature of PAN is around $250^{\circ} \mathrm{C}$ because of the presence of ester comonomers (VAc), which need higher activation energy for cyclization (Ouyang et al. 2008). The lignin shows a very strong and broad exothermic profile, which is mainly due to the intermolecular crosslinking and repolymerization of the smaller molecules formed via bond cleavage (Oroumei et al. 2015). The initiation temperature of lignin is around $190^{\circ} \mathrm{C}$ due to its higher thermoreactivity. The maxima of lignin/PAN blend PFs moves to the lower temperature range compared to that of pure PAN PFs. The initiation temperature of the lignin/PAN blend PFs decreased to about $190^{\circ} \mathrm{C}$, which is close to that of lignin.

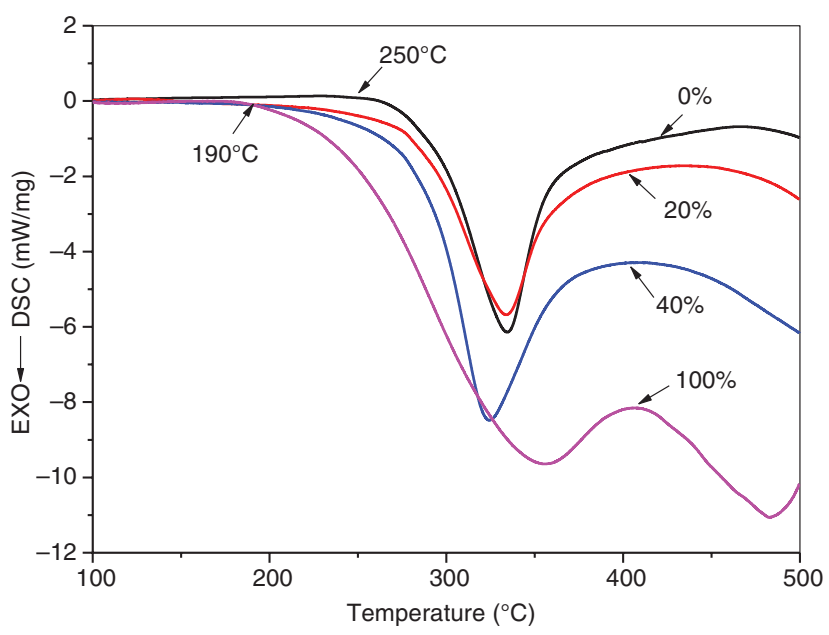

Figure 5: DSC curves of lignin and lignin/PAN blend PFs. 
With increasing lignin contents, the exothermic band of the lignin/PAN blend PFs becomes stronger. Probably, the carbonyl/carboxyl groups of lignin are able to initiate the cyclization reaction by ionic mechanism at lower temperatures (Ouyang et al. 2008). As a result, thermal stabilization can be carried out at lower temperatures, which can contribute to lowering the production costs.

Figure 6a shows the density of PFs and thermo-stabilized fibers. The densities of these materials increase with increasing lignin contents because of the higher density of lignin. The density differences between PFs and thermostabilized fibers were calculated (see Figure 6a). As is visible, the density differences significantly increased at higher lignin contents.

Figure 6b shows the FTIR spectra of thermo-stabilized fibers. The extent of the cyclization reaction was calculated using the equation $E_{c}=A_{1595} / A_{2243}$ (Ouyang et al. 2008) and the data are listed in Table 3. As not otherwise expected, $E_{c}$ values increase significantly with increasing lignin contents.

Because of the large fiber diameter, the mechanical properties of the CFs are not satisfactory. The tensile
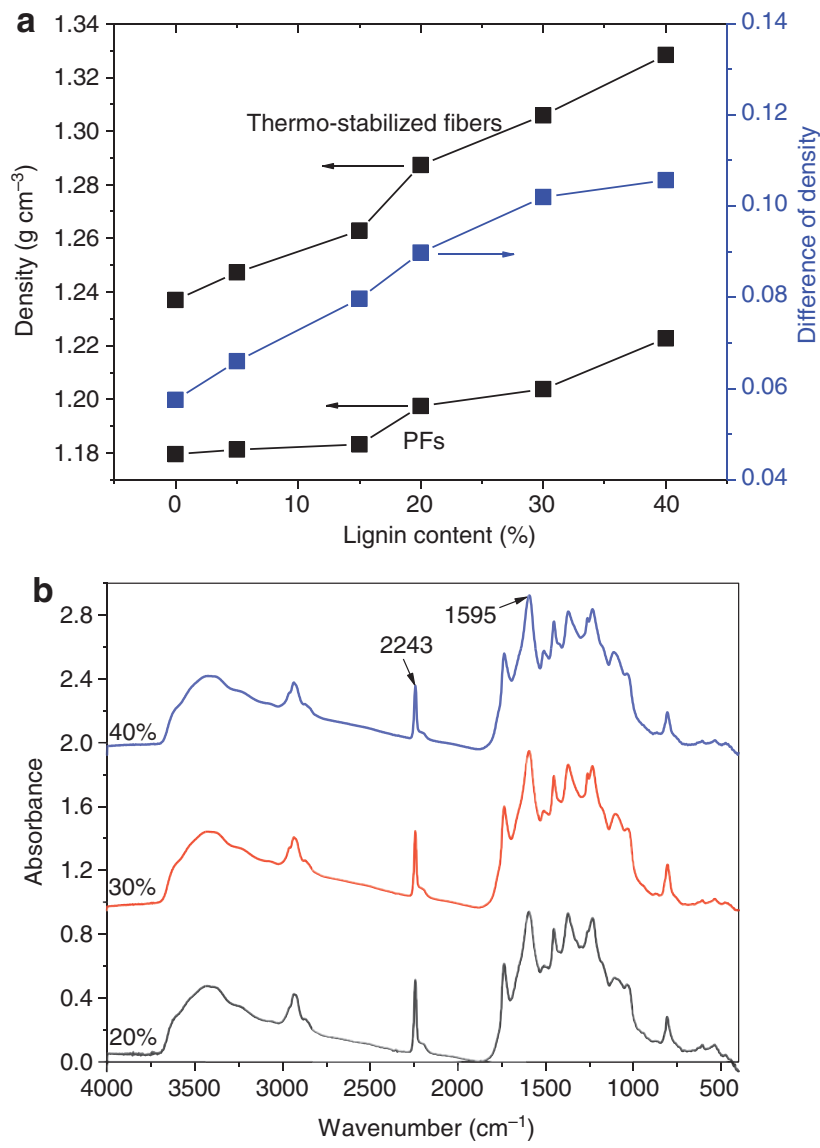

Figure 6: (a) Density and (b) FTIR spectra of thermo-stabilized fibers.
Table 3: The extent of cyclization reaction ( $E_{c}$ values) of thermo-stabilized fibers.

\begin{tabular}{llll}
\hline & \multicolumn{3}{c}{ Lignin content in thermo-stabilized fibers } \\
\cline { 2 - 4 } & $\mathbf{2 0 \%}$ & $\mathbf{3 0 \%}$ & $\mathbf{4 0 \%}$ \\
\hline$E_{c}$ value & 0.97 & 1.17 & 1.56 \\
\hline
\end{tabular}

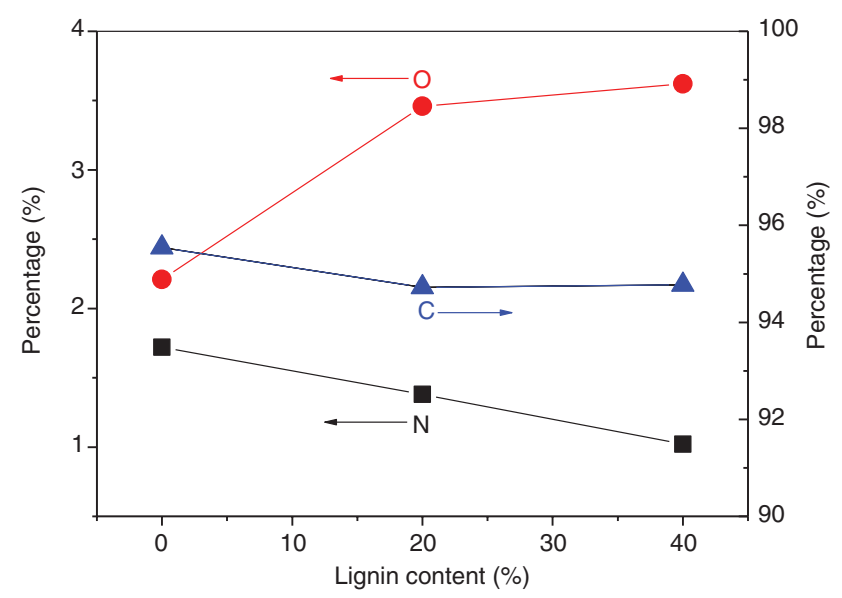

Figure 7: Element content of CFs.

strength of the CFs is only 300-500 MPa, and the tensile modulus is below $100 \mathrm{GPa}$. Figure 7 shows the element content of CFs with different lignin contents. According to the elemental composition of lignin and PAN, the oxygen content increases significantly, and the contents of nitrogen and carbon element decrease slightly with increasing lignin contents up to $20 \%$. At higher lignin contents, the elemental composition changes further in the same direction, but the carbon content remains around 95\%. It is not exactly clear why the carbon content is so stable.

\section{Conclusions}

The viscosity of the spinning solutions and the structure of the PFs were significantly affected by the lignin content in the blends, while the changes are closely related to the intermolecular forces in the spinning solution. Lignin plays a similar role as the carboxylic acid comonomer and results in a significant decrease of the initiation temperature of the exothermic maximum in the DSC profiles. The thermal stabilization of the lignin/PAN blend PFs was significantly promoted by lignin, which contributes to lowering the production cost of CFs. The morphology of the CFs is inherited from the PFs. When the lignin content was increased to 
$30-40 \%$, macro-voids became visible in the center of the CFs. Thirty percent of the blended lignin content seems to be an insurmountable bottleneck for the preparation of CFs based on lignin/PAN blends. A positive finding is the high carbon content $(\approx 95 \%)$ of the CFs, and thus lignin could be used for blends, if its concentration is not above $20 \%$.

Author contributions: All the authors have accepted responsibility for the entire content of this submitted manuscript and approved submission.

Research funding: National Key Research and Development Plan of China (2016YFB0101702) is gratefully acknowledged for the financial support.

Employment or leadership: None declared.

Honorarium: None declared.

\section{References}

Arbab, S., Mirbaha, H., Zeinolebadi, A., Nourpanah, P. (2014) Indicators for evaluation of progress in thermal stabilization reactions of polyacrylonitrile fibers. J. Appl. Polym. Sci. 131:77-81.

Boeriu, C.G., Bravo, D., Gosselink, R.J.A., Dam, J.E.G.V. (2004) Characterisation of structure-dependent functional properties of lignin with infrared spectroscopy. Ind. Crops Prod. 20:205-218.

Chatterjee, S., Saito, T. (2015) Lignin-derived advanced carbon materials. ChemSusChem. 8:3941-3958.

Doi, M., Edwards, S.F. (1978) Dynamics of concentrated polymer systems. Part 2. - Molecular motion under flow. J. Chem. Soc. Faraday Trans. 74:1802-1817.

Dong, X., Lu, C., Zhou, P., Zhang, S., Wang, L., Li, D. (2015) Polyacrylonitrile/lignin sulfonate blend fiber for low-cost carbon fiber. RSC Adv. 5:42259-42265.

Foston, M., Nunnery, G.A., Meng, X., Sun, Q., Baker, F.S., Ragauskas, A. (2013) NMR a critical tool to study the production of carbon fiber from lignin. Carbon 52:65-73.

Huang, X. (2009) Fabrication and properties of carbon fibers. Materials 2:2369-2403.

Huang, Y., Zhao, G. (2016) Preparation and characterization of activated carbon fibers from liquefied wood by $\mathrm{KOH}$ activation. Holzforschung 70:195-202.

Huang, Y., Yu, W., Zhao, G. (2018) Activated carbon fiber from liquefied wood and polyvinyl butyral as an additive for production of flexible all-carbon yarn supercapacitors. Holzforschung 72:367-374.

Husman, G. Development and commercialization of a novel low-cost carbon fiber. Presentation at 2013 DOE Hydrogen and Fuel Cells Program and Vehicle Technologies Program Annual Merit Review and Peer Evaluation Meeting, 2012.

Husman, G. Development and commercialization of a novel low-cost carbon fiber. Presentation at 2014 DOE Hydrogen and Fuel Cells Program and Vehicle Technologies Program Annual Merit Review and Peer Evaluation Meeting, 2014.

Li, Q., Serem, W.K., Dai, W., Yue, Y., Naik, M.T., Xie, S., Karki, P., Liu, Li., Sue, H., Liang, H., Zhou, F., Yuan, J. (2017) Molecular weight and uniformity define the mechanical performance of lignin-based carbon fiber. J. Mater. Chem. A.

5:12740-12746.

Liu, H.C., Chien, A.-T., Newcomb, B.A., Liu, Y., Kumar, S. (2015) Processing, structure, and properties of lignin- and CNTincorporated polyacrylonitrile-based carbon fibers. ACS Sustain. Chem. Eng. 3:1943-1954.

Mainka, H., Täger, O., Körner, E., Hilfert, L., Busse, S., Edelmann, F.T., Herrmann, A.S. (2015) Lignin - an alternative precursor for sustainable and cost-effective automotive carbon fiber. J. Mat. Res. Technol. 4:283-296.

Miller, G.C., Yu, J., Joseph, R.M., Choudhury, S.R., Mecham, S.J., Baird, D.G., Bortner, M., Norris, R.E., Paulauskas, F.L., Riffle, J.S. (2017) Melt-spinnable polyacrylonitrile copolymer precursors for carbon fibers. Polymer 126:87-95.

Nowak, A.P., Hagberg, J., Leijonmarck, S., Schweinebarth, H., Baker, D., Uhlin, A., Tomani, P., Lindbergh, G. (2018) Lignin-based carbon fibers for renewable and multifunctional lithium-ion battery electrodes. Holzforschung 72:81-90.

Oroumei, A., Fox, B., Naebe, M. (2015) Thermal and rheological characteristics of biobased carbon fiber precursor derived from low molecular weight organosolv lignin. ACS Sustain. Chem. Eng. 3:758-769.

Ouyang, Q., Cheng, L., Wang, H., Li, K. (2008) Mechanism and kinetics of the stabilization reactions of itaconic acid-modified polyacrylonitrile. Polym. Degrad. Stab. 93:1415-1421.

Park, C.W., Youe, W.J., Han, S.Y., Kim, Y.S., Lee, S.H. (2017) Characteristics of carbon nanofibers produced from lignin/polyacrylonitrile (PAN)/kraft lignin-g-PAN copolymer blends electrospun nanofibers. Holzforschung 71:743-750.

Ragauskas, A.J., Beckham, G.T., Biddy, M.J., Chandra, R., Chen, F., Davis, M.F., Davison, B.H., Dixon, R.A., Gilna, P., Keller, M., Langan, P., Naskar, A.K., Saddler, J.N., Tschaplinski, T.J., Tuskan, G.A., Wyman, C.E. (2014) Lignin valorization: improving lignin processing in the biorefinery. Science 344:1246843.

Seydibeyoglu, M.O. (2012) A novel partially biobased PAN-lignin blend as a potential carbon fiber precursor. J. Biomed. Biotechnol. 2012:598324.

Shan, J., Guan, Y., Zheng, Q., Han, J., Liu, Q., Pu, Z. (2009) Application of urea/H2O2 activation-oxidation system in degradation of PVA and desizing of polyester/cotton fabric. J. Appl. Polym. Sci. 113:860-867.

Thunga, M., Chen, K., Grewell, D., Kessler, M.R. (2014) Bio-renewable precursor fibers from lignin/polylactide blends for conversion to carbon fibers. Carbon 68:159-166.

Wang, S., Li, Y., Xiang, H., Zhou, Z., Chang, T., Zhu, M. (2015) Low cost carbon fibers from bio-renewable lignin/poly(lactic acid) (PLA) blends. Compos. Sci. Technol. 119:20-25.

Warren, C.D., Paulauskas, F.L., Baker, F.S., Eberle, C.C., Naskar, A. (2009) Development of commodity grade, lower cost carbon fiber-commercial applications. SAMPE J. 45:24-36.

Xia, K., Ouyang, Q., Chen, Y., Wang, X., Qian, X., Wang, L. (2016) Preparation and characterization of lignosulfonate-acrylonitrile copolymer as a novel carbon fiber precursor. ACS Sustain. Chem. Eng. 4:159-168.

Ye, W., Li, X., Luo, J., Wang, X., Sun, R. (2017) Lignin as a green reductant and morphology directing agent in the fabrication of 3D graphene-based composites for high-performance supercapacitors. Ind. Crops Prod. 109:410-419. 
Yoo, S.H., Park, S., Park, Y., Lee, D., Joh, H.-I., Shin, I., Lee, S. (2017) Facile method to fabricate carbon fibers from textile-grade polyacrylonitrile fibers based on electron-beam irradiation and its effect on the subsequent thermal stabilization process. Carbon 118:106-113.

Youe, W.J., Lee, S.M., Lee, S.S., Lee, S.H., Kim, Y.S. (2016) Characterization of carbon nanofiber mats produced from electrospun lignin-g-polyacrylonitrile copolymer. Int. J. Biol. Macromol. 82:497-504.

Supplementary Material: The online version of this article offers supplementary material (https://doi.org/10.1515/hf-2017-0191). 